\title{
Simplifying Bleeding Risk Assessment in Heart Failure
}

\author{
Kotaro Nochioka, MD, PhD
}

$\mathbf{P}$ atients with heart failure (HF) are not only at increased risk of thromboembolic events but also heightened risk of bleeding. ${ }^{1,2} \mathrm{~A}$ previous report has shown that beyond age, peripheral arterial disease, diabetes, hypercholesterolemia, hypertension, smoking antiplatelet agents or oral anticoagulants, HF is itself a risk

\section{Article p 147}

factor of bleeding, requiring a new stratification strategy for identification of individuals with high bleeding risk (HBR) in HF. ${ }^{2}$

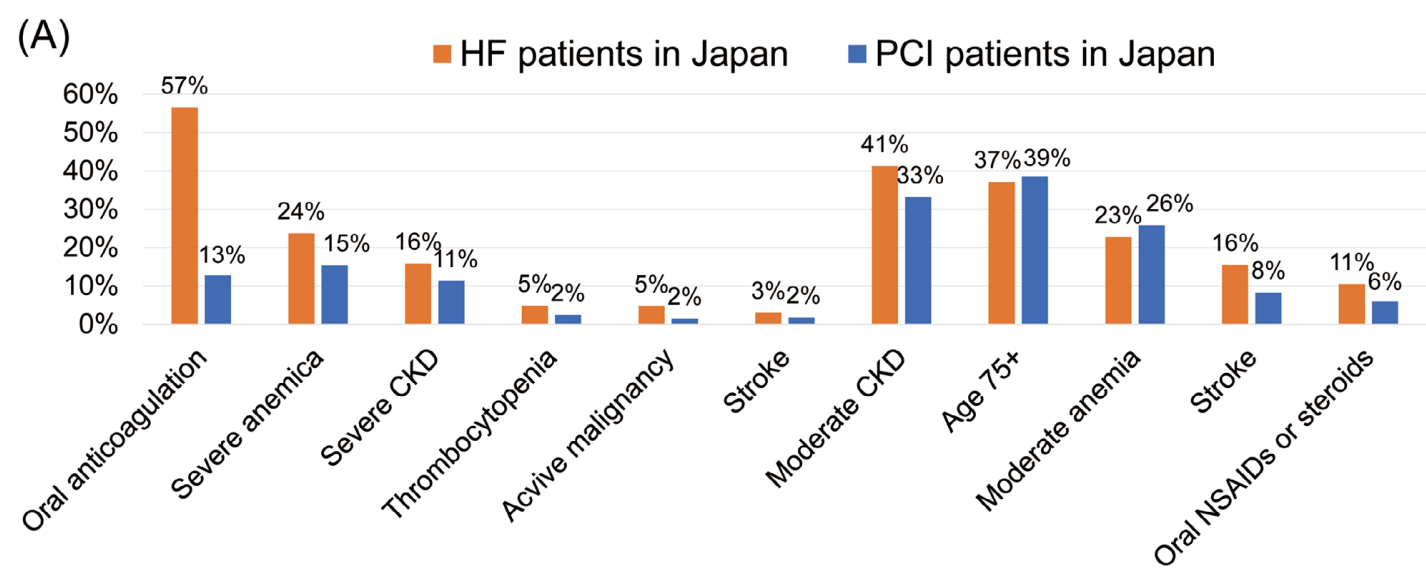

(B)

- Hazard ratio $(95 \% \mathrm{Cl})$ for bleeding in $\mathrm{HF}$

Simplified ARC-HBR

$\geq 2$ Minor criteria

1 Major criteria

$\geq 2$ Major criteria

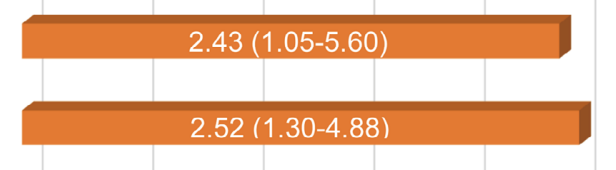

$3.34(1.70-6.56)$

$0.5 \quad 1$

1.5

2

2.5

3

3.5

Figure. (A) Comparison of the prevalence of the simplified ARC-HBR criteria in $\mathrm{HF}^{\mathbf{7}}$ and $\mathrm{PCl}^{\mathbf{5}}$ patients in Japan. (B) Coexistence of multiple ARC-HBR criteria and bleeding risk in HF patients. ARC-HBR, Academic Research Consortium for High Bleeding Risk; $\mathrm{Cl}$, confidence interval; CKD, chronic kidney disease; HF, heart failure; NSAIDs, nonsteroidal anti-inflammatory drugs; PCI, percutaneous coronary intervention.

The opinions expressed in this article are not necessarily those of the editors or of the Japanese Circulation Society.

Received November 3, 2021; accepted November 3, 2021; J-STAGE Advance Publication released online November 26, 2021

Department of Cardiovascular Medicine, Tohoku University Graduate School of Medicine, Clinical Research, Innovation and Education Center, Tohoku University Hospital, Sendai, Japan

Mailing address: Kotaro Nochioka, MD, PhD, MPH, Department of Cardiovascular Medicine, Tohoku University Graduate School of Medicine, Clinical Research, Innovation and Education Center, Tohoku University Hospital, 1-1 Seiryo-machi, Aoba-ku, Sendai 980-8574, Japan. E-mail: nochioka@cardio.med.tohoku.ac.jp

All rights are reserved to the Japanese Circulation Society. For permissions, please e-mail: cj@j-circ.or.jp

ISSN-1346-9843 
In 2018, as a collaboration among leading research organizations, regulatory authorities, and physician-scientists from the USA, Europe and Japan, the Academic Research Consortium for High Bleeding Risk (ARC-HBR) launched a project to identify and manage patients with HBR undergoing percutaneous coronary intervention (PCI), given that the lack of standardization in defining this population limits trial design, data interpretation, and clinical decision-making. ${ }^{3}$ The ARC-HBR proposed a new definition consisting of 17 major and minor criteria to standardize HBR. Following this proposal, studies reported the generality of the definition in real-world settings from PCI registries. ${ }^{4,5}$ Furthermore, Cao et al reported that coexistence of multiple ARC-HBR criteria provided additive prognostic value not only for bleeding but also for thrombotic events, including all-cause death in patients after PCI. ${ }^{5}$ However, Miura et al recently reported that 6 (prior bleeding and transfusion defined in both major and minor criteria, chronic bleeding diathesis, liver cirrhosis with portal hypertension, nondeferrable major surgery on dual antiplatelet therapy, and prior trauma or surgery within 30 days) of 17 criteria were observed in $<1 \%$ of patients in daily clinical practice settings, and proposed a simplified ARC-HBR definition generated by excluding low-frequency criteria from the original definition for easier identification of HBR patients. ${ }^{6}$ They also provided the prognostic value of the simplified ARC-HBR definition, based on the area under curves of the original and simplified definitions for prediction of major bleeding being comparable $(0.598$ [95\% CI 055-0.64] and 0.600 [95\% CI 0.55-0.64], $\mathrm{P}=0.08$ ) in patients after PCI, highlighting the clinical utility of the simplified definition. ${ }^{6}$

The study by Sato and colleagues, ${ }^{7}$ the results of which are published in this issue of the Journal, is the first to expand the clinical application of the simplified ARCHBR definition to HF patients. Using the database from a single site including 2,437 patients hospitalized for $\mathrm{HF}$, they observed that $>80 \%$ of the HF patients had HBR, emphasizing importance of HBR in HF. Furthermore, using Fine-Gray competing regression analysis considering all-cause death as the competing factor, ${ }^{8}$ these authors show that the simplified ARC-HBR definition clearly identified HF patients who experienced bleeding events (Figure). They observed no interactions between the simplified ARC-HBR definition and other important factors such as past medical history of coronary artery disease, use of antiplatelet agents, and HF severity, implying the association between the definition and bleeding events is independent of patient status. Of note, they report that coexistence of multiple ARC-HBR criteria informed additive prognostic value: the simplified definition also classified the patients at higher risk for rehospitalization for HF, all-cause death, cardiac death, and major adverse cardiac and cerebrovascular events, suggesting other clinical implications of the simplified definition.

As the authors acknowledge in the limitations, this study was conducted in a single center and needs validation in other HF cohorts. In addition, they do not provide comparative data in terms of feasibility and prognostic value between the original and simplified ARC-HBR definitions, which may limit assessment of the accuracy of risk prediction using the simplified ARC-HBR. However, simplifying the definition would make it feasible to apply risk stratification in daily practice. In conclusion, this study highlights the clinical utility of the simplified ARC-HBR criteria for predicting a high risk of bleeding events in HF patients, but calls for further studies to optimize the management of $\mathrm{HF}$.

\section{Disclosure}

None.

\section{References}

1. Uretsky BF, Thygesen K, Armstrong PW, Cleland JG, Horowitz JD, Massie BM, et al. Acute coronary findings at autopsy in heart failure patients with sudden death: Results from the Assessment of Treatment with Lisinopril and Survival (ATLAS) Trial. Circulation 2000; 102: 611-616.

2. Ducrocq G, Wallace JS, Baron G, Ravaud P, Alberts MJ, Wilson PWM, et al. Risk score to predict serious bleeding in stable outpatients with or at risk of atherothrombosis. Eur Heart $J$ 2010; 31: $1257-1265$.

3. Urban P, Mehran R, Colleran R, Angiolillo DJ, Byrne RA, Capodanno D, et al. Defining high bleeding risk in patients undergoing percutaneous coronary intervention: A consensus document from the Academic Research Consortium for High Bleeding Risk. Eur Heart J 2019; 40: 2632-2653.

4. Ueki Y, Bär S, Losdat S, Otsuka T, Zanchin C, Zanchin T, et al. Validation of the Academic Research Consortium for High Bleeding Risk (ARC-HBR) criteria in patients undergoing percutaneous coronary intervention and comparison with contemporary bleeding risk scores. EuroIntervention 2020; 16: $371-379$.

5. Cao D, Mehran R, Dangas G, Baber U, Sartori S, Chandiramani $\mathrm{R}$, et al. Validation of the Academic Research Consortium High Bleeding Risk Definition in contemporary PCI patients. $J$ Am Coll Cardiol 2020; 75: 2711-2722.

6. Miura K, Shimada T, Ohya M, Murai R, Amano H, Kubo S, et al. Prevalence of the Academic Research Consortium for High Bleeding Risk Criteria and prognostic value of a simplified definition. Circ J 2020; 84: 1560-1567.

7. Sato Y, Yoshihisa A, Takeishi R, Ohara H, Sugawara Y, Ichijo Y, et al. Simplified Academic Research Consortium for High Bleeding Risk (ARC-HBR) definition predicts bleeding events in patients with heart failure. Circ J 2022; 86: 147-155.

8. Fine JP, Gray RJ. A proportional hazards model for the subdistribution of a competing risk. J Am Stat Assoc 1999; 94: 496509 . 